\title{
A Systematic Literature Review on Service Description Methods
}

\author{
Abelneh Y. Teka, Nelly Condori-Fernandez, Brahmananda Sapkota \\ University of Twente, \\ Enschede, The Netherlands. \\ a.y.teka@student.utwente.nl, \{n.condorifernandez,b.sapkota\}@utwente.nl
}

\begin{abstract}
Context and Motivation] As a result of recent trends in enhancing Service Oriented Requirement Engineering activities, a number of service description methods have been proposed for describing services. The availability of different service description methods can give developers a range of options to choose from so that they can have an appropriate description method that fits best their services. [Question/problem] But there is neither holistic information on service description methods nor a clear understanding of the strengths and weaknesses of each service description method. The aim of this paper is to identify problems of service descriptions that have been researched so far, and the techniques or methods available to tackle these problems. [Principle ideas/results] Thus, to gather this relevant information available in the literature, a systematic review was conducted. A total of 191 articles were examined, of which 24 articles focus on service description related concepts. The results show that, despite the recent efforts in describing the nonfunctional requirements of services through approaches like semantic annotations and policy attachments, there is still a lot to do in enhancing the description of quality aspects of services. Furthermore, this study reveals that a negligible effort is given to the description of consumer oriented services. [Contribution] This paper identifies and analyzes the current service description methods that exist in the literature and explains the pros and cons inherent to these methods
\end{abstract}

Keywords: systematic review, service description, service specification, functional, non-functional requirements

\section{Introduction}

A successful Requirements Engineering (RE) process involves understanding the needs of customers, and other stakeholders; understanding the contexts in which the to-be-developed software will be used; modeling, analyzing, negotiating, and documenting the stakeholders' requirements; validating that the documented requirements match the negotiated requirements; and managing requirements evolution [1]. Service Oriented Requirements Engineering (SORE) shares with these activities, but some of them are conducted in a different way. The most remarkable difference is that service and workflow discovery has a very significant role in SORE as part of the requirement elicitation and analysis activities [8],[39]. SORE focuses on 
determining requirements of systems which are going to be developed in a serviceoriented manner [2].

Although RE is a key part in software development process; there is still a lack of well-established and widely accepted RE methods even in the commonly used system development approaches like Object Oriented approaches [3]. The same is true in the newly Service Oriented Computing (SOC) paradigm, where one of the consequences, associated with this lack of appropriate RE techniques, is the absence of accurate service descriptions ${ }^{1}$, which will affect other SOC activities like service discovery and service composition [5], [6].

This lack in accurate service description is manifested by the presence of gaps between the specifications of requirements of a system and the service oriented description of the system. The gap is due to the difference in focuses of the two systems, i.e. Requirement engineering is primarily concerned with goals and requirements while service descriptions are mainly about technical operations and bindings [37].

Thus currently, researchers are enthusiastically producing new techniques in order to cover this gap. And the development of new approaches for describing services is not a problem by itself; in fact, it gives an opportunity for practitioners to have a range of choices to use in specific situations. The real problem is the lack of holistic information on available methods and techniques along with their respective strengths and weakness. Though scarce studies that allow gaining this holistic view of existing methods have been carried out [13], [10], a comprehensive analysis covering different aspects of the available service description methods is still missing.

This paper aims to analyze the current service description methods that exist in the literature, by identifying pros and cons of these methods. To do this, a Systematic Literature Review (SLR) is performed based on the guidelines suggested by Kitchenham et al. [11]. We decided to conduct a SLR instead of a Mapping Study because our research goes beyond of identifying the quantity and type of research and results available within a research area [40].

The remaining parts of this paper are organized as follow: Section 2 introduces the main issue of our review, service description, from a SORE viewpoint. Section 3 focuses on the methodology used in conducting the research. Section 4 presents the results of this review. Finally, section 5 concludes the paper.

\section{Background: Service description}

Requirement engineering in SOC plays a vital role in identifying and specifying service requirements that have been defined through service level agreements (SLAs) [12]. SORE focuses on identification, specification and analysis of requirements. But the specification of SLAs need different approaches in requirement engineering as there are a number of activities in SORE that are not available in the traditional RE

1 A Service description comprises a service specification and, if available, some service additional Information. A service specification is usually defined by the service developer and may include both functional and non-functional information [39] 
activities. Examples of such activities include the requirements elicitation from service description and service discovery processes.

Utilization of a service based application involves a number of entities playing different roles. Among these entities, service providers, service repositories and service consumers are the key stakeholders that SORE is focusing on. Service providers publish their service descriptions on Service repositories and service consumers use these descriptions for discovering and binding to services in order to utilize them [2]. This means, service consumers need information about services available at repositories so that they can discover and ultimately utilize it. Thus, it is important to have an expressive service description that enables service consumers to decide which services are best suited for satisfying their requirements.

Currently services are described by service description languages like Web Service Description Language (WSDL), but service orientation itself needs its own requirement engineering activities, since a service described in terms of operations and bindings may not be enough to specify the desired goals and domain assumptions of stakeholders [41].

This does not mean available service description methods are all unable to specify requirements as they are supposed to do so. In fact there are considerable number of emerging approaches ([7], [16], [17], [27]) targeted at closing the gap between technical service description techniques and the common RE specifications.

\section{Review Methodology}

The major steps taken to conduct this literature study are taken from the guidelines proposed by Kitchenham et. al [11], which are discussed in the following subsections:

\subsection{Defining the research questions}

As we mentioned in the Section 1, this paper focuses on existing service description methods, associated problems and possible approaches to tackle these problems. In particular, we aim at answering the following research questions:

RQ1. What are the existing service description methods reported in literature?

RQ2. What are the problems faced during service description process as reported in the literature?

RQ3. What are the strengths and weaknesses of these service description methods?

\subsection{Search and selection process}

The principal source used while searching relevant papers was Scopus (www.scopus.com). As Scopus provides access to well-known bibliographicdatabases like IEEE Explore, SpringerLinks, ACM digital library etc. at the same place, it was fruitful using it as a search engine for the search process. 
In the search process, the identification of our search string was carried out in an iterative way. We started with a number of combinations of search terms like: "Service Description" AND "Requirement specification", "Service Specification" AND "requirement specification", "service oriented architecture" AND "requirement engineering". As thousands of articles were retrieved, we restricted these preliminary search results by limiting the subject area to computer science and discarding papers published earlier than $2002^{2}$. Doing so helped us to discard irrelevant articles much easily from other areas (e.g. economics). The list of search terms was adapted several times and the search was re-run with the new terms. (See Table 1).

\section{Table 1: Search hits from Scopus}

\begin{tabular}{|l|l|l|l|l|}
\hline Search Term & $\begin{array}{l}\text { Number } \\
\text { of first } \\
\text { hits }\end{array}$ & $\begin{array}{l}\text { Restriction } \\
\text { to computer } \\
\text { science } \\
\text { subject area }\end{array}$ & $\begin{array}{l}\text { Restriction to } \\
\text { publication } \\
\text { date year } \\
2002 \quad \text { and } \\
\text { above }\end{array}$ & $\begin{array}{l}\text { Restriction } \\
\text { to } \\
\text { conference } \\
\text { papers and } \\
\text { articles }\end{array}$ \\
\hline $\begin{array}{c}\text { "service AND } \\
\text { architecture" } \\
\text { "requirement engineering" }\end{array}$ & 572 & 432 & 408 & 366 \\
\hline $\begin{array}{l}\text { "Service Description" AND } \\
\text { "Requirement } \\
\text { specification", }\end{array}$ & 38 & 29 & 29 & 28 \\
\hline $\begin{array}{l}\text { "Service Specification" } \\
\text { AND "Requirement } \\
\text { specification" }\end{array}$ & 3,518 & 1,514 & 1,187 & 1112 \\
\hline
\end{tabular}

After an iterative refinement, the search string used was the following: (("service oriented architecture") OR SOA) AND (("service description" OR "service specification") AND ("requirement specification")).

A total of 191 articles were retrieved from this search string. From these articles, a further refinement was carried out. 11 studies were identified by reading their abstract; 3 studies were identified by reading the introduction part; and 8 studies were identified by reading whole article. At this phase the authors observe that the approaches discussed in [29] and [31] are similar and merged to one approach making the relevant service description methods count to be 21 articles.

While we were reviewing these 21 articles, 3 more ([10], [15], [31]) were identified as relevant for our study. These articles were incorporated in the relevant list, thereby ending up in 24 articles selected for the study presented in this paper.

\subsection{Study quality assessment and data collection}

For studying the quality assessment, a qualitative assessing was carried out within the selection process. We consider an article as relevant for our review whether it

\footnotetext{
${ }^{2}$ It is starting time of Service Oriented Architecture
} 
reports "enough" information to answer our main research questions. In addition, although the $70 \%$ of the articles were refereed, the criteria by number of citation was not also considered in order to do a filter of our 24 articles, since a good number of the articles were published in the last year (2010).

Each of the 24 papers selected was analyzed, by identifying 1) the problem to be solved by the service description method proposed, 2) the technique(s)/language used to tackle the problem, 3) their strengths and weaknesses, and 4) the approach employed to evaluate or validate the respective description method.

\section{Review results}

\subsection{RQ1: Service Description Methods.}

The literature study reveals that there has been a significant effort in improving requirement engineering practices for SOA. For instance, as shown in Figure 1, from the 24 service description methods, 19 of them are published in and after 2007.

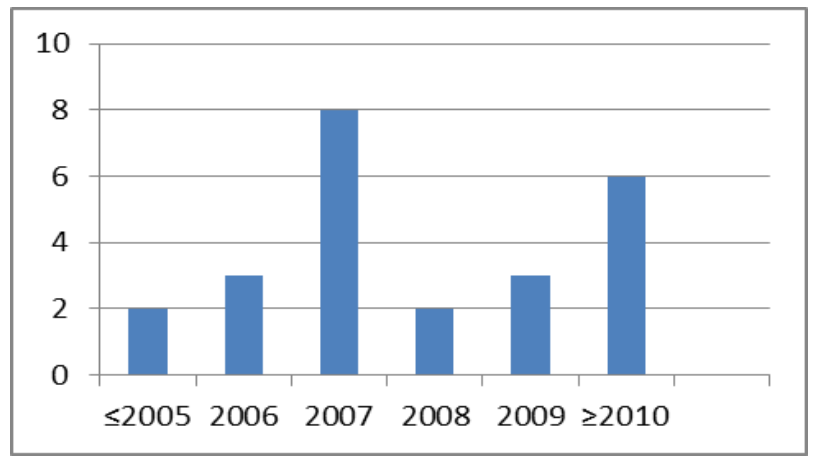

Fig. 1. Frequency of newly emerged description methods by year.

As the service description methods found are diverse and this broad variety makes it difficult to classify them based on certain criteria, we consider to use three dimensions with the purpose of facilitating the analysis of each one of the 24 service description methods. Figure 2 shows these three dimensions: Representation (syntactic, semantic), Content (Functional requirements, Non-functional requirements, additional information), and Perspective (business, operational and technical). Table 4 (See Appendix) shows an overview of these 24 methods according to these three dimensions. 


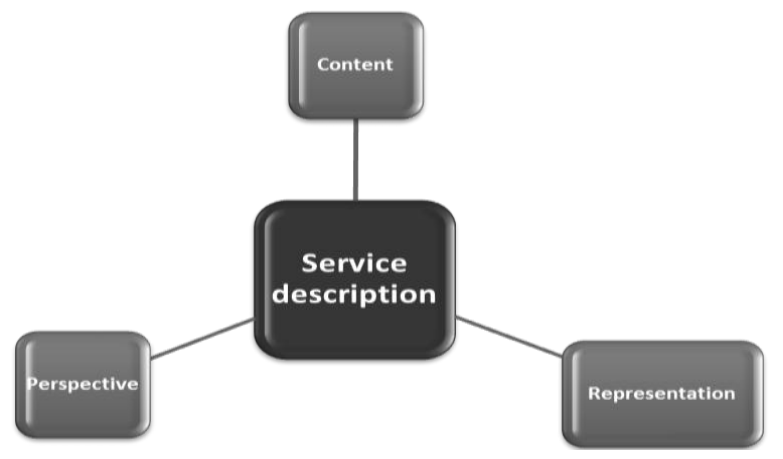

Fig. 2. Dimensions used to analyze service description methods

Syntax Based vs Semantic Based Methods. Syntax based service descriptions are the most commonly employed description methods up to date. These methods hide what is going on inside the service and expose the necessary input and output values of the service interfaces. Syntax based service description employs techniques based on languages like WSDL to expose the interface of the service and other service description mechanisms like Web Service Level Agreement (WSLA).

The syntax based description methods that use WSDL as service description language at least as their base in describing services are [16], [20], [24], [28].

Semantic based service descriptions are emerging approaches that describe service based on various forms of meanings like ontology annotation and Context information based methods [10].Semantic based descriptions can be an extension of services described in WSDL [20], but can also be entirely dependent on ontology for describing services [14].

The extension of WSDL approaches adds additional semantic description to WSDL components by using annotations while the pure semantic approaches use modifications of Web Ontology Language (OWL) for specifying the functionalities of a service and the associated inputs and outputs. OWL is not the only language used in semantic service descriptions. Its predecessor DARPA Agent Markup Language (DAML) can be used for describing services as a process not as a one shoot activity while retaining the semantic meanings [32].

Functional vs Non Functional Requirements Description Methods. The functional requirements of services can be described in terms of syntax based, behavior description based and semantic description based approaches [10]. Though most service description techniques are intended for describing the functional requirements of services [5], [16], [28], [29], [35] ; there are a considerable number of approached that aim in giving emphasis to the non-functional requirements too[18], [21].

The presence of the non-functional requirements like quality, cost, legal issues, etc. will definitely add more options for customers to choose the right service for their requirements. Some of these methods are helpful in describing even frequently changing Quality of Services (QoSs) that helps service users in selecting services that matches their requirements [21]. Contrary to this distinction between functional and 
non-functional requirements descriptions, there are also more promising approaches which consider both the functional and non-functional requirements [20], [30].

Business Process - Technology Mapping approaches. SOC is targeted for developing enterprise applications based on autonomous services [16]. Previous trends in developing such applications were technology oriented. Under such approach, services are usually defined in terms of technical functionalities. These approaches describe services based on the various operations defined at their interfaces to be invoked at different port types [4], [16], [36].

The most important thing to consider here is that applications are usually developed to achieve some kind of business goal. Such business goals are generally realized/represented in terms of some business processes expressed in business processes modeling languages like be BPEL or BPMN. In SOA approach, these business processes are realized by service based applications which are represented in terms of technical service development process so that the service based application can assist in achieving the business goals. Business Process - Technology Mapping approaches help in a better alignment of business processes to services based applications. From the 24 service description methods found in the study [7], [16], [17], [27] were targeted at a better alignment of business - technology mappings.

Using a unified service description language is one of the approaches discovered for such enhanced alignment [7]. This approach proposes model-based description of services from business operational and technical perspectives. Another candidate approach discovered to handle such an issue is formulating business specifications to include SOA application specification [17]. Such approaches are designed to improve the poor role played by the current service description methods in business-software mapping mechanisms.

Model Based Service Description Approaches. Requirements engineers in SOA usually use model driven approaches to specify the requirements of the services. Among the 24 service description methods found in the review, [7], [24], [25], [30], [26] use various model based approaches to describe services. There are modeling approaches like SMMA (Single Model Multiple Analysis) that can help even in generating codes from the models alone [15]. PSML-S (Process Specification and Modeling Language) is a typical language used for implementing SMMA approach. An alternative language that can be used in specifying services is BPEL4WS which is mainly used in defining the flow and coordination between service components [29].

There are also formal model based techniques for describing services though they still need more time to mature enough to be applicable in service oriented applications [15]. But efforts to improve the applicability of these formal methods are already taking place. ForSel (Formal Service description language) is a typical example of such efforts. ForSel describes services by describing the necessary functionalities in terms of finite or infinite reactions [5]. Using calculus of communication systems (CCS) to model behavior of services is also another approach discovered for describing services [33]. 
Service Descriptions for Adaptable Services. In today's competitive business environment, business goals and requirements tend to change regularly. Consequently, applications that support in realization of these requirements need to be updated regularly. And for effective dynamic realizations of business processes, dynamic composition of services is essential.

Moreover, for dynamic service composition, presence of dynamic service description techniques is important. From the 21 service description methods found in the review [9], [14], [23], [28] target at providing descriptions for such dynamic and adaptable services.

An interesting approach dynamic service description approach found during the study is the adaptation of situation awareness in service specification. In support of this, a new extension of OWL-S, named as SAW-OWL-S, was developed to enable services to realize their business context [9]. Related to these, a new approach named Extended Web Service Agreement was proposed to enable renegotiations of SLAs that can help in modifying QoSs while the service is being provided [28]. This approach uses a new element named modifiable service level objective that can be modified at run time.

Along with the growth in popularity of SOA, service based applications is being used not only in large business process contexts but also in small scale businesses and even in our day to day personal activities. Developing services for such processes is usually cumbersome as the developer may not have even a concrete clue on the customer requirements. Such kind of problems can be tackled by letting consumers to specify and publish their requirements and then developers can design services based on clients' requirements [29].

We also found methods for describing services based on mathematical/formal specifications. For instance, in order to describe services developed for reactive systems, a method named Formal Service description is proposed in [5]. This approach specifies services as a composition of precondition, triggering event and the system reaction. Furthermore, an additional method named Formal specification of data aspects of Web services [35] was proposed for ensuring that customers' requirements are still satisfied during change of services implementations by service providers.

\subsection{RQ2: Problem faced in describing services.}

The second step taken in reviewing the selected 24 papers was to find what type of problem is the proposed methods are aiming to solve. We found that majority of the papers focus on problems related to lack of describing semantic meaning and the Quality of Service (QoS) properties of services.

We also observe that there is a wide gap in services realized from business process perspective and from the software engineering perspective. There are also problems associated with services described in terms of producer centric approaches. Table 2 shows the major problems discovered in the literature study. 
Table 2. Problems addressed by the 24 service description methods.

\begin{tabular}{ll}
\hline Problem & References \\
\hline Lack of Semantic in syntactic Descriptions and & {$[4],[9],[14],[18-26]$} \\
failure in describing QoS. & \\
Gap between business oriented and IT oriented & {$[7],[16],[17],[27]$} \\
Service Realizations. & \\
Lack of dynamic adaptability and situation & {$[9],[14],[23],[28]$} \\
awareness & {$[29],[31]$} \\
Producer only centric approaches & {$[5]$} \\
Imprecise Requirements Specification & {$[32],[33]$} \\
Lack of Behavior Description & \\
\hline
\end{tabular}

Lack of QoS Descriptions. It has been noted that even though message oriented description techniques, like WSDL [14], are most popular ones; they have their own limitations. WSDL describes services in terms of various operations defined at interfaces. These descriptions are published on the publicly accessible service registries. Users will discover such services by matching the inputs and outputs of operations at these interfaces [4]. Such approaches in service descriptions employ syntactic matching in service discovery. A typical problem associated with this is the keywords used in service description may fail in describing all the relevant services as keywords can have different synonyms [10]. It is possible to use wildcards during service discovery to alleviate such situation but doing so is not the best solution as it will result in many irrelevant service for the service consumer.

As nonfunctional requirements are also integral parts of services, QoS should also need to be described just like the functional requirements [4]. Unfortunately, the syntax based service description techniques like WSDL fail in realizing this crucial part of a service description.

Gap between Business Service and IT Service. Services are designed to automate a certain business process. So they can be realized from two perspectives: business services and technical services. Business services are concerned with the end to end delivery and an outcome of the process while most of the current service description methods fail in describing the details of these processes as they specify services in terms of a mere input and output operations [7]. This introduces difficulties in aligning business-software realizations [17]. If such details are not realized in the service implementations, there is no guarantee that the developed services will fully realize the business requirements.

Lack of Adaptability and Situation Awareness. Consumers will start utilizing services once they agreed with the providers and establish a Service Level Agreements (SLAs). Sometimes, after starting to use the agreed services, the service users' requirements may change, which may lead to the change in SLAs altogether. These changes are in fact a highly probable situation to arise in today's dynamic business environment. 
Additionally, changes can be requested not only by the service consumers but also by the service providers. Unfortunately, the current service description methods are not capable of handling changes in service level agreements once it is set in to operation [28].

Service composition is also one of the vital activities in SOA as it can provide new functionality by composing existing services. But unfortunately, services described based on syntactic approach fails in composing services dynamically, i.e., service composition needs human involvement [14]. In addition, there is also a considerable lack of support in incorporating context and situation awareness of the service environment during service description. Such lack in situation description will result in less flexible service design [9].

Lack of Consumer Oriented Service Description. As SOC is used for more and more applications, applications for personal uses are being developed based on services. Even end-users with no technical background are creating their own web applications [8]. Such users may not know the exact requirements of their applications. In addition, service based applications for individuals face problems associated with the broad variety of customer needs. Developing services that can satisfy such varying needs is difficult, if not impossible, for service developers [29].

\subsection{RQ3: Strengths and Weakness of Current Service Description Methods.}

Each group of service description approaches shown in Table 3 and Table 4 (See Appendix) has their own strengths and weakness. In this section, we present the strong and weak points of these methods. At this point, we would like to remind that some methods are designed to tackle the limitation of another method. This can result in a situation where one method's limitation is some other method's good feature.

Syntax based approaches. Syntactic service description methods are the most frequently used service description methods to date [32]. These methods describe a service by explaining the values that are entering and leaving the services without specifying the details of the internal structure of the services. These methods separate the interface of their services from the actual bindings necessary to access services [16]. This will enhance the modularity of the services as they are no longer tied to one implementation. Furthermore, as these methods expose the public interfaces only, developers can use any implementation technique as long as it can provide the desired operations at the interfaces [4].

The huge problem associated with describing services in terms of their syntactic signature is the complete lack in describing QoS [14] . As nonfunctional requirements are the integral parts of any service, the inability of syntax based description methods to describe these crucial parts of services is a severe drawback that forces developers to seek for other approaches for describing services [4].

The inability to describe QoS is not the only problem faced by developers using syntactic description methods. As there is no semantic representation of services in these approaches, they can also result in a low precision service discovery results 
[21]. In addition, the lack in semantic meaning of inputs and outputs makes it impossible for a complete automation of finding and invoking required services [14].

Semantic based approaches. Semantic description of services has a wide range of advantages as they can provide meaning to service descriptions. A semantic rich description can describe not only the functional requirements but also the nonfunctional requirements of services too [20]. Such availability of semantic meaning to services will enable users to select the right service for their business process when they are faced with vague syntax based descriptions [23].

Though semantic based approaches are successful especially in mitigating major weakness of syntax based service description methods, they also come with their own limitation. Their first limitation is associated with the complexity of ontological concepts and relation between them. It is usually cumbersome to use these complex concepts by both service providers and consumers to avoid semantic heterogeneity [10]. Context aware service development is also one of the emerging approaches in service based applications industry. But let alone the syntax based approaches, ontology based description techniques like OWL-S also lack appropriate mechanisms in formal expression of context and situations [9].

\section{Functional requirement based description. The functional requirements} descriptions of services are crucial in ensuring users that the services they are going to utilize will satisfy their demands. Web service repositories like UDDI usually store information about the functionality of services [10]. As these functionalities are the primary concerns of the majority service users, service description techniques based on these approaches are preferred by users with their primary concern, which is the automation of the main business process.

The limitations of theses functional requirement descriptions are mainly related with their lack in describing the quality and dynamic aspects of the services [19], [20]. These limitations are directly related to the limitations of syntax based approaches as the functional requirement description methods use the syntax based approaches in describing services.

Model based descriptions. Most of the SORE activities employ one or more types of model -driven approaches. One of the benefits of using such models is that once there is a well-established set of core models it will be relatively easier to analyze and maintain custom built models based on these core service models. Furthermore, it is possible to use these models for automatic code generation [8]. Modeling of services in using formal methods like Calculus of Communicating Systems (CCS) will also enable for description of the behavior of services that were not available in syntax based approaches [33]. The prominent limitation of describing services by using models is the complexity involved in the formal specification of services. To avoid such problems, service providers should have adequate knowledge on modeling languages like CCS and automata.

Finally, we also reviewed the efforts aimed in validating or evaluating the existing service description methods. The observed result was not encouraging since 10 out of 24 description methods use simple examples only for showing their validity, and 8 out of the remaining 14 papers do not specify any validation or evaluation approach at all. 
This clearly indicates the need for more research to evaluate more rigorously in reallife settings. The complete list of service description methods identified in this study can be referred in Appendix (See Table 3).

\subsection{Limitations of this Review.}

The main limitations of this review are bias in the selection of relevant articles and a data extraction bias. Our search string was limited only to computer science subject area due to "service" term is used by other disciplines (e.g. medicine, economics, social sciences, engineering). Besides, it was also necessary to limit year of publication to 2002 to increase the precision search. However, these both limitations could be affecting our recall search.

Another limitation is related to the accuracy of data extraction; several articles lacked sufficient information regarding the dimensions considered for describing services. For example, some articles do not precisely state the type of content described, whether it is functional or nonfunctional (e.g. [6]) description or they do not precisely state the type of representation, whether it is semantic and syntactic (e.g. [29]) description. There is, therefore, a possibility that the extraction process may have resulted in some inaccuracy in the data.

Moreover, with respect to the synthesis of our findings, we recognize that it could have been carried out in a more systematic way, if we had used some of the tools for synthetizing qualitative studies (e.g. EPPI-reviewer $4^{3}$ ). However at the moment of conducting the present review we did not have a software license available to use the tool.

\section{Conclusions and Future Work}

Service descriptions are one of the major activities included in SORE as it is a crucial prerequisite to service discovery process. This paper presents problems faced in describing services, 24 service description methods discovered from literature study and the strengths and weakness of these service description methods. The result of the study was presented according to the major categories of service description methods currently employed or proposed to be employed.

Though WSDL is the most widely used service description language, it comes with its own limitation: It fails in describing nonfunctional requirements of services which are of course crucial parts of services [4]. Such and related problems along with the possible solutions are discussed in Section 4.

The study shows that there is a considerable limitation in describing QoS nonfunctional requirements of services despite the presence of some efforts in describing these quality attributes. A possible approach observed to handle this lack of support for describing QoS in service description is to integrate syntactic based descriptions like WSDL and ontology based descriptions like OWL-S. As WSDL

\footnotetext{
${ }^{3}$ http://eppi.ioe.ac.uk/cms/
} 
descriptions are quite extensible, adding OWL annotations can be fruitful in describing both the functional and QoS aspects of services.

Considering the recent trends in developing service oriented applications targeted mainly for personal use, the authors observe a negligible amount of effort in handling requirement specifications of these user-centric applications. There is also a significant difference in business process specification and how the current service implementation technologies realize and describe these business processes.

On the other hand, from this SLR, we think that a list of possible combinations of service description methods could have resulted to yield much better description approach, thereby increasing the expressiveness of the specification document. In fact we have observed such possibilities. For instance Syntax based service descriptions work nicely only if specification documents are available to explain the details of the capabilities of the service as well as the conditions necessary for using the service [22]. But if such specification document is not available, verbose service description methods ([19]) come in to play. Syntax based and semantic based service description methods can be combined to provide rich service description approaches [20]. But this and other possibilities of combining two or more description methods will be part of our future work.

In addition, we will be also focused on investigating further approaches aimed at enhancing the current efforts in describing QoS. As the quality attributes are crucial in specifying service capabilities, more study on service descriptions focusing on QoS will be quite relevant.

We also plan to integrate this study with existing service description and service discovery mechanisms to create a holistic view of the basic activities in SORE.

\section{Acknowledgments}

This work was supported in part by the EU Marie Curie Fellowship Grant 50911302 PIEF-2010. Authors would like also to thank the anonymous reviewers, and colleagues Klaas Sikel and Zortnitza Bakalova for their valuable comments for improvement.

\section{References}

1. B. Cheng, and J. M. Atlee (2007). Research Directions in Requirements Engineering. Requirements Engineering, 285-303.

2. M. Galster and E. Bucherer, "Towards Requirements Engineering in a Service-Oriented Environment-Extending the SOA Interaction Triangle," in Proceedings of the International Conference on Computational Intelligence for Modelling Control \& Automation, 2008, pp. 1099-1104.

3. Davis AM and Hickey AM, "A New Paradigmfor Planning and Evaluating Requirements Engineering Research," 2004.

4. M. Papazoglou, Web Services: Principles and Technology, 1st ed. Prentice Hall, 2007.

5. J. Hartmann, S. Rittmann, D. Wild, and P. Scholz, "Formal incremental requirements specification of service-oriented automotive software systems," in Proceedings of the 
Second IEEE International Symposium on Service-Oriented System Engineering, 2006, pp. 130-133.

6. D. Edmond, A. H. M. Hofstede, and J. O'sullivan, "Service Description: A survey of the general nature of services," University of Queensland, vol. 12, pp. 117 - 133.

7. J. Cardoso, A. Barros, N. May, and U. Kylau, "Towards a unified service description language for the internet of services: Requirements and first developments," in Proceedings of the IEEE 7th International Conference on Services Computing, 2010, pp. 602-609.

8. W. T. Tsai, Z. Jin, P. Wang, and B. Wu, "Requirement Engineering in Service-Oriented System Engineering," in Proceedings of the IEEE International Conference on e-Business Engineering, 2007, pp. 661-668.

9. S. S. Yau and J. Liu, "Incorporating situation awareness in service specifications," in Proceedings of the Ninth IEEE International Symposium on Object-Oriented Real-Time Distributed Computing, 2006, pp. 287-294.

10. D. A. D'Mello and V. S. Ananthanarayana, "A review of dynamic web service description and discovery techniques," in Proceedings of the 1st International Conference on Integrated Intelligent Computing, 2010, pp. 246-251.

11. Kitchenham, Barbara, "Procedures for Performing Systematic Reviews", Technical Report, TR/SE-0401, Keele University, 2004.

12. S. Lichtenstein, L. Nguyen, and A. Hunter, "Issues in IT service-oriented requirements engineering," [Online]. Available: http://www.deakin.edu.au/dro/view/DU:30005308. [Accessed: 01-Jun-2011].

13. Q. Gu and P. Lago, "Service Identification Methods: A Systematic Literature Review," in Towards a Service-Based Internet, vol. 6481, E. Nitto and R. Yahyapour, Eds., Springer, 2010, pp. 37-50.

14. D. Martin et al., "Bringing semantics to web services: The OWL-S approach," in Lecture Notes in Computer Science, 2005, vol. 3387, pp. 26-42.

15. W.-tek Tsai, Y. Chen, and C. Fan, "PESOI: Process Embedded Service-Oriented Architecture *."

16. M. Rychlý and P. Weiss, "Modeling of service oriented architecture from business process to service realisation," in Proceedings of the 3rd International Conference on Evaluation of Novel Approaches to Software Engineering, 2008, pp. 140-146.

17. B. Shishkov, J. L. G. Dietz, and M. van Sinderen, "Closing the Business-Application GAP in SOA challenges and solution directions," in 2nd International Conference on Software and Data Technologies, Proceedings, 2007, vol. SE, pp. 333-336.

18. A. A. A. Slimane, M. K. Pinheiro, and C. Souveyet, "Goal reasoning for quality elicitation in the ISOA approach," in Proceedings of the 3rd International Conference on Research Challenges in Information Science, 2009, pp. 39-48.

19. M. Stefanovic, M. Matijević, M. Erić, and V. Simic, "Method of design and specification of web services based on quality system documentation," Information Systems Frontiers, vol. 11, no. 1, pp. 75-86, 2009.

20. Q. Qiu and Q. Xiong, An ontology for semantic web services, vol. 4782. 2007.

21. K. Kritikos and D. Plexousakis, "Requirements for QoS-based Web service description and discovery," IEEE Transactions on Services Computing, vol. 2, no. 4, pp. 320-337, 2009.

22. H. Pfeffer, D. Linner, C. Jacob, I. Radusch, and S. Steglich, "Towards light-weight semantic descriptions for decentralized service-oriented systems," in International Conference on Semantic Computing, 2007, pp. 295-303.

23. E. Sirin, J. Hendler, and B. Parsia, "Semi-automatic Composition of Web Services using Semantic Descriptions," In Web Services: Modeling, Architecture and Infrastructure Workshop, 2003, p. 17--24, 2002. 
24. P. Bocciarelli and A. D'Ambrogio, "A model-driven method for describing and predicting the reliability of composite services," Software \& Systems Modeling, vol. 10, no. 2, pp. 265-280, Feb. 2010.

25. A. Di Marco and A. Sabetta, "Model-based dynamic QoS-driven service composition," in ACM International Conference Proceeding Series, 2010.

26. P. Fornasier, J. Webber, and I. Gorton, "Soya: A Programming Model and Runtime Environment for Component Composition Using SSDL," in Component-Based Software Engineering, vol. 4608, H. W. Schmidt, I. Crnkovic, G. T. Heineman, and J. A. Stafford, Eds., Springer, 2007, pp. 227-241.

27. C. Rolland, M. Kirsch-Pinheiro, and C. Souveyet, "An intentional approach to service engineering," IEEE Transactions on Services Computing, vol. 3, no. 4, pp. 292-305, 2010.

28. G. Di Modica, V. Regalbuto, O. Tomarchio, and L. Vita, "Enabling re-negotiations of SLA by extending the WS-Agreement specification," in Proceedings of the IEEE International Conference on Services Computing, 2007, pp. 248-251.

29. W. T. Tsai, X. Bingnan, R. Paul, H. Qian, and C. Yinong, "Global software enterprise: A new software constructing architecture," in CEC/EEE 2006 Joint Conferences, 2006.

30. N. C. Narendra and K. Ponnalagu, "Variation-Oriented Requirements Analysis (VORA)," in Proceedings of the IEEE Congress on Services, SERVICES 2007, 2007, pp. 159-166.

31. W. T. Tsai, B. Xiao, R. A. Paul, and Y. Chen, "Consumer-centric service-oriented architecture: A new approach," in Proceedings of the Fourth IEEE Workshop on Software Technology for Future Embedded and Ubiquitous Systems, and the Second International Workshop on Collaborative Computing, Integration, and Assur. 2006, pp. 175-180.

32. M. Klein, B. König-Ries, and P. Obreiter, Stepwise refinable service descriptions: Adapting DAML-S to staged service trading, vol. 2910. 2003.

33. B. Yun, J. Yan, and M. Liu, "Behavior-Based Web Services Matchmaking," in Proceedings of the 2008 IFIP International Conference on Network and Parallel Computing, 2008, pp. 483-487.

34. M. V. Zelkowitz and D. Wallace, "Experimental validation in software engineering" Information and Software Technology, vol. 39, no. 11, pp. 735-743, 1997.

35. I. Saleh, G. Kulczycki, and M. B. Blake, "Formal specification and verification of datacentric service composition" in Proceedings of the IEEE 8th International Conference on Web Services, 2010, pp. 131-138.

36. P. Bocciarelli and A. D'Ambrogio, "A model-driven method for describing and predicting the reliability of composite services," Software and Systems Modeling, vol. 10, no. 2, pp. 265-280, 2011.

37. B. Verlaine, Y. Dubois, I. J. Jureta, and S. Faulkner, "Towards automated alignment of Web Services to requirements," in Web and Requirements Engineering (WeRE), 2010 First International Workshop on the, 2010, pp. 5-12.

38. W. Hummer, P. Leitner, and S. Dustdar, "SEPL-a domain-specific language and execution environment for protocols of stateful Web services" 2011.

39. M. P. Papazoglou, K. Pohl, M. Parkin, A. Metzger: Service Research Challenges and Solutions for the Future Internet - S-Cube - Towards Engineering, Managing and Adapting Service-Based Systems, Springer 2010.

40. K. Petersen, R. Feldt, M. Shahid, M. Mattsson, "Systematic Mapping Studies in Software Engineering", 12th International Conference on Evaluation and Assessment in Software Engineering (EASE), Department of Informatics, University of Bari, Italy, June 2008.

41. B. Verlaine, I. J. Jureta, and S. Faulkner, "Towards conceptual foundations of requirements engineering for services," in 2011 Fifth International Conference on Research Challenges in Information Science (RCIS), 2011, pp. 1-11. 


\section{Appendix}

Table 3. An overview of existing service description methods

\begin{tabular}{|c|c|c|c|}
\hline Ref. & Method name & $\begin{array}{l}\text { Technique/ Language } \\
\text { used }\end{array}$ & $\begin{array}{l}\text { Validation/evaluative } \\
\text { approach }\end{array}$ \\
\hline M1 [18] & $\begin{array}{l}\text { Intentional Service, for } \\
\text { Quality of Service }\end{array}$ & $\begin{array}{l}\text { Text based, no } \\
\text { specific language } \\
\text { employed }\end{array}$ & Quality model \\
\hline M2 [7] & $\begin{array}{l}\text { Modeling business and } \\
\text { technical services }\end{array}$ & USDL & Testing \\
\hline M3 [28] & $\begin{array}{l}\text { Extended WS } \\
\text { agreement }\end{array}$ & WSLA & Not Specified \\
\hline M4 [19] & $\begin{array}{l}\text { Specifications based on } \\
\text { quality system } \\
\text { documentation }\end{array}$ & Documentation based & Questionnaire \\
\hline M5 [5] & $\begin{array}{l}\text { Formal service } \\
\text { description }\end{array}$ & ForSeL Calculus & Case study on progress \\
\hline M6 [16] & $\begin{array}{l}\text { Modeling of business } \\
\text { process to service } \\
\text { diagrams }\end{array}$ & $\begin{array}{l}\text { WSDL like operation } \\
\text { specification }\end{array}$ & Example \\
\hline M7 [17] & $\begin{array}{l}\text { SOA driven } \\
\text { specification }\end{array}$ & $\begin{array}{l}\text { SOA-driven business- } \\
\text { software mapping }\end{array}$ & Not Specified \\
\hline M8 [29] & $\begin{array}{l}\text { Consumer Oriented } \\
\text { SOA }\end{array}$ & $\begin{array}{l}\text { Tools like PSML-S } \\
\text { and BPEL }\end{array}$ & Example \\
\hline M9 [30] & $\begin{array}{l}\text { Variation Oriented } \\
\text { requirement analysis }\end{array}$ & $\begin{array}{l}\text { VORA tractability } \\
\text { model }\end{array}$ & Example \\
\hline M10 [27] & $\begin{array}{l}\text { Intentional approach for } \\
\text { service description }\end{array}$ & $\begin{array}{l}\text { Intentional Service } \\
\text { Modeling }\end{array}$ & Example \\
\hline M11 [38] & Service protocol & SEPL & Testing \\
\hline M12 [24] & $\begin{array}{l}\text { Model base approach } \\
\text { for describing QOS }\end{array}$ & Q-WSDL & Example \\
\hline M13 [33] & $\begin{array}{l}\text { Behavior based service } \\
\text { description }\end{array}$ & $\begin{array}{l}\text { Formal description } \\
\text { based on CCS }\end{array}$ & Example \\
\hline M14 [25] & $\begin{array}{l}\text { Model-based dynamic } \\
\text { QoS-driven service } \\
\text { composition }\end{array}$ & SMART & Not specified \\
\hline M15 [26] & SOYA & SSDL & Not specified \\
\hline M16 [20] & $\begin{array}{l}\text { Semantic annotation for } \\
\text { WSDL }\end{array}$ & $\begin{array}{l}\text { Annotation of WSDL } \\
\text { components }\end{array}$ & Example \\
\hline M17 [9] & $\begin{array}{l}\text { Situation aware service } \\
\text { based systems }\end{array}$ & $\begin{array}{l}\text { SAW-OWL-S } \\
\text { (Extension of OWL-S) }\end{array}$ & Example \\
\hline M18 [4] & WS-Policy attachment & $\begin{array}{l}\text { WS Policy and WS } \\
\text { agreement }\end{array}$ & Example \\
\hline M19 [35] & $\begin{array}{l}\text { Formal Specification of } \\
\text { data aspects of web } \\
\text { services }\end{array}$ & $\begin{array}{l}\text { Formal representation } \\
\text { of contracts }\end{array}$ & $\begin{array}{l}\text { Formal verification: } \\
\text { Symbolic reasoning }\end{array}$ \\
\hline M20 [21] & Ontology for QoS & $\mathrm{OWL}-\mathrm{Q}$ & Not Specified \\
\hline M21 [14] & $\begin{array}{l}\text { Semantic for Web } \\
\text { services }\end{array}$ & OWL-S & Example \\
\hline M22 [22] & Semantics for Service & Distributed semantic & Not Specified \\
\hline
\end{tabular}




\begin{tabular}{llll}
\hline Ref. & Method name & $\begin{array}{l}\text { Technique/ Language } \\
\text { used }\end{array}$ & $\begin{array}{l}\text { Validation/evaluative } \\
\text { approach }\end{array}$ \\
\hline M23 [23] & $\begin{array}{l}\text { Descriptions } \\
\text { Semi-automatic } \\
\text { semantic descriptions } \\
\text { for web services } \\
\text { Refining service } \\
\text { Descriptions }\end{array}$ & $\begin{array}{l}\text { trees. } \\
\text { DAML }\end{array}$ & $\begin{array}{l}\text { PAML-S } \\
\text { (DAML for services) }\end{array}$ \\
\hline
\end{tabular}

Table 4. Service description Methods

\begin{tabular}{|c|c|c|c|c|c|c|c|c|c|}
\hline & \multicolumn{3}{|c|}{ Content } & \multicolumn{3}{|c|}{ Representation } & \multicolumn{3}{|c|}{ Perspective } \\
\hline Method & $\mathbf{F u}$ & NonF & Other & Verb & Synt & Sema & Buss & Oper & Tech \\
\hline M1 & & $\mathrm{X}$ & & $\mathrm{X}$ & & & $\mathrm{X}$ & & \\
\hline M2 & $X$ & $X$ & & & $X$ & & $X$ & $X$ & $\mathrm{X}$ \\
\hline M3 & $X$ & & & & & & & & \\
\hline M4 & $\mathrm{X}$ & $\mathrm{X}$ & & $\mathrm{X}$ & & & $\mathrm{X}$ & & \\
\hline M5 & $X$ & & & & $\mathrm{X}$ & $X$ & & & \\
\hline M6 & $X$ & & & & $X$ & & $X$ & & \\
\hline M7 & $X$ & & & & & & $\mathrm{X}$ & & \\
\hline M8 & $\mathrm{X}$ & & X & & & & $\mathrm{X}$ & & \\
\hline M9 & $X$ & $X$ & & & & & & & \\
\hline M10 & $X$ & & & & & & $X$ & & \\
\hline M11 & $X$ & & & & & & & & \\
\hline M12 & & $\mathrm{X}$ & & & & & & & \\
\hline M13 & $X$ & & & & & & & & \\
\hline M14 & & X & & & & & & & \\
\hline M15 & $\mathrm{X}$ & & & & & $X$ & & & $X$ \\
\hline M16 & $X$ & $\mathrm{X}$ & & & & $\mathrm{X}$ & & & \\
\hline M17 & & $X$ & X & & & & & & \\
\hline M18 & & X & & & & & & & \\
\hline M19 & $\mathrm{X}$ & & & & & & & & \\
\hline M20 & $X$ & X & & & & $\mathrm{X}$ & & & \\
\hline M21 & & $X$ & & & $X$ & & & & \\
\hline M22 & $X$ & & & & & $X$ & & & \\
\hline M23 & $\mathrm{X}$ & & $X$ & & & $X$ & & & \\
\hline M24 & $X$ & & & & $X$ & & & & \\
\hline
\end{tabular}

\section{Legend:}

Fu: Functional Requirements NonF: Non Functional Requirements Other: Additional information Verb: Verbose

Synt: Syntactic
Sema: Semantic

Buss: Bussiness Oper: Operational Tech: Technical 